\title{
ARTICLE
}

\section{A transcriptome analysis of two grapevine populations segregating for tendril phyllotaxy}

\author{
Jie Arro ${ }^{1}$, Jose Cuenca ${ }^{1}$, Yingzhen Yang ${ }^{1}$, Zhenchang Liang ${ }^{2}$, Peter Cousins ${ }^{3}$ and Gan-Yuan Zhong ${ }^{1}$
}

The shoot structure of cultivated grapevine Vitis vinifera L. typically exhibits a three-node modular repetitive pattern, two sequential leaf-opposed tendrils followed by a tendril-free node. In this study, we investigated the molecular basis of this pattern by characterizing differentially expressed genes in 10 bulk samples of young tendril tissue from two grapevine populations showing segregation of mutant or wild-type shoot/tendril phyllotaxy. One population was the selfed progeny and the other one, an outcrossed progeny of a Vitis hybrid, 'Roger's Red'. We analyzed 13375 expressed genes and carried out in-depth analyses of 324 of them, which were differentially expressed with a minimum of 1.5-fold changes between the mutant and wild-type bulk samples in both selfed and cross populations. A significant portion of these genes were direct cis-binding targets of 14 transcription factor families that were themselves differentially expressed. Network-based dependency analysis further revealed that most of the significantly rewired connections among the 10 most connected hub genes involved at least one transcription factor. TCP3 and MYB12, which were known important for plant-form development, were among these transcription factors. More importantly, TCP3 and MYB12 were found in this study to be involved in regulating the lignin gene $P R X 52$, which is important to plant-form development. A further support evidence for the roles of TCP3-MYB12-PRX52 in contributing to tendril phyllotaxy was the findings of two other lignin-related genes uniquely expressed in the mutant phyllotaxy background.

Horticulture Research (2017) 4, 17032; doi:10.1038/hortres.2017.32; Published online 12 July 2017

\section{INTRODUCTION}

Grapevine (Vitis spp.) is one of the oldest domesticated fruit crops in the world. Grown mostly for wine since antiquity, grapevine berries and their derivatives are also found in familiar consumer products such as table grapes, raisins, juice, jelly and dietary supplements due to recently known beneficial effect of its antioxidant, resveratrol. ${ }^{1}$ Understandably, until recently, the wine industry has driven a significant portion of the research on berry quality and wine-related traits such as color and juice compositional profile, as well as disease resistance and abiotic stress tolerance. $^{2}$ In contrast, research needs in vine growth and development, and even yield have received much less attention with fewer number of research studies reported, even fewer than that related to vineyard management. ${ }^{3}$ Part of the reason that impedes a full understanding of grapevine developmental processes is the complex biology of grapevine itself. ${ }^{4,5}$ Grapevine takes more than a year to complete a growth cycle, one of the longest life cycles among the cultivated crops; its developmental stages involve many complex processes such as lateral bud formation, dormancy and burst, and obtaining reliable phenotypic data are challenging given its size, growing habits, viticulture practice and extensive interactions with external environments. In spite of these, some substantial knowledge has been gained about the vegetative and reproductive growth and development of grapevines, although the knowledge accumulation has rather been incremental. ${ }^{3,6,7}$

Unlike annual herbaceous plants, the growth and development of grapevine is unique and complex. ${ }^{8}$ The switch from juvenile to adult phase in Vitaceae, which encompass grapevines (Vitis) and
900 member-species in other 15 genera, ${ }^{9}$ is signified by the appearance of the first tendril together with the switch from spiral to alternate leaf arrangement. ${ }^{4}$ From then on, growth proceeds with the shoot apical meristem (SAM) in the main branch's apex continually giving rise to both leaf primordia and an uncommitted primordia called anlagen. ${ }^{10,11}$ Depending on the genetic and environmental cues, this uncommitted primordia can become one of the two homologous organs in Vitaceae, tendril or inflorescence. This feature is unique to Vitaceae. Any inflorescences formed in the vegetative phase of the first year, however, will undergo dormancy only to resume from the lateral buds in the following spring towards reproductive growth. This monopodial growth pattern, which spans a life cycle of at least 2 years characterized by indeterminate growth and lateral branching, defines the shoot architecture typical of Vitaceae. ${ }^{12}$ Thus, to some degree, growth and development in grapevine is inextricably linked to understanding its shoot organogenesis, shoot architecture and reproductive development. ${ }^{4}$

Except for a few, most species in the family Vitaceae possess tendrils and are known for their climbing habit.,13 Tendrils are tactile string-like motile organs adapted for grappling and supporting the growing vine as it climbs atop canopies for maximum light interception in the wild. Tendrils are, as recognized by Darwin, adaptive morphological innovation in plants. Indeed, convergent adaptive evolution in other plants such as Fabaceae, Cucurbitaceae and Smilacaeae gave rise to tendrils from modified leaflets, shoots and stipules, respectively. ${ }^{14}$ In Vitaceae, however, tendril occupies a more significant biological role due to its close organogenetic relationship with

${ }^{1}$ USDA-Agricultural Research Service, Grape Genetics Research Unit, Geneva, NY 14456, USA; ${ }^{2}$ Beijing Key Laboratory of Grape Science and Enology and Key Laboratory of Plant Resource, Institute of Botany, the Chinese Academy of Sciences, Beijing 100093, People's Republic of China and ${ }^{3}$ E\&J Gallo Winery, Modesto, CA 95353, USA. 
inflorescences. Aside from their roles in movement and support, tendrils express inflorescence genes, ${ }^{15-17}$ and some of which were thought to control the miRNA-mediated phase change to flowering. ${ }^{3}$ Gerrath et al. ${ }^{12}$ recognized a modular three-node vegetative growth pattern in vine shoot growth and development, which in combination with presence and position of the latent buds, is a species-specific signature in Vitaceae. In the most widely cultivated Vitis species, Vitis vinifera, tendrils normally appear in repeating units of three nodes, opposite each leaf in two sequential nodes and then a skip on the third. However, in the North American wild Vitis species, Vitis labrusca, continuous presence of tendrils on nodes was observed. ${ }^{18}$

Some Vitis hybrids and cultivars were reported to show disruption in the regularity of the modular, three-node order of two successive tendrils followed by a skip. The $V$. vinifera var. 'Grenache' and 'Syrah' showed variable degrees of disrupted tendril phyllotaxy especially at the basal nodes, ${ }^{19}$ and that development in secondary axes were different depending on the position in the three-node module. ${ }^{20}$ Cousins et al. ${ }^{21,22}$ selfed a hybrid with disrupted tendril distribution pattern and also crossed it with a vine with normal tendril distribution. They observed segregation of the trait in both progeny populations. Taken together, this suggests that the three-node phyllotaxy is under genetic control. Cousins and Zhong ${ }^{23}$ proposed that the mutant type was likely controlled by two epistatic loci with supplementary dominant-gene action.

We report here an RNA-Seq differential expression analysis of bulk samples from two populations segregating for tendril phyllotaxy. Of the 13375 expressed genes, we identified 324 differentially expressed genes (DEGs) in both populations. These DEGs include many transcription factor (TF) families involved in a myriad of growth and development processes. Our results shed light on the molecular processes involved in the tendril development, helping develop further understanding of the genetic control of reproductive traits and related plant architecture in grapevine.

\section{MATERIALS AND METHODS}

Plant material

Two segregating populations were used in this study (Table 1). One was derived from the cross of PC04206-36 $\times$ Roger's Red. The female parent, PC04206-36, is a nematode-resistant breeding selection from the USDAAgricultural Research Service Grape Rootstock Improvement Program in Geneva, NY, USA. ${ }^{23}$ It shows a normal pattern of tendril phyllotaxy (a threenode pattern in which tendrils are present on the opposite sides of leaves in the first two sequential nodes but no tendril is on the third node). The male parent, Roger's Red, is an ornamental vine valued for its deep red autumn foliage. Molecular marker data confirmed that Roger's Red is a natural hybrid between $V$. californica and a red-fleshed $V$. vinifera, Alicante

Table 1. RNA-Seq bulk samples and their population background phenotypes and other properties

\begin{tabular}{lllcc}
\hline $\begin{array}{l}\text { Bulk sample } \\
\text { ID }\end{array}$ & Population & Phenotype & $\begin{array}{c}\text { No. of individual } \\
\text { progeny vines }\end{array}$ & $\begin{array}{c}\text { Library } \\
\text { size }(G b)\end{array}$ \\
\hline P65.m.2 & P65-crossed Mutant & 8 & 2.98 \\
P65.m.3 & P65-crossed & Mutant & 9 & 4.40 \\
P65.m.4 & P65-crossed Mutant & 11 & 5.93 \\
P65.n.1 & P65-crossed Wild-type & 9 & 4.25 \\
P65.n.2 & P65-crossed Wild-type & 9 & 1.67 \\
P96.m.1 & P96-selfed Mutant & 4 & 3.45 \\
P96.m.2 & P96-selfed & Mutant & 4 & 2.98 \\
P96.m.3 & P96-selfed Mutant & 5 & 4.23 \\
P96.n.4 & P96-selfed Wild-type & 4 & 3.05 \\
P96.n.5 & P96-selfed Wild-type & 4 & 1.99 \\
\hline
\end{tabular}

Bouchet. ${ }^{24}$ Roger's Red has a high frequency of an interrupted or mutant pattern of tendril phyllotaxy (Cousins, unpublished data). This cross population, hereon referred to as ' $P 65$ ', had a total of 175 progeny lines with $140(80 \%)$ normal progenies and $35(20 \%)$ mutant (Table 2). The other population used in this study, named as 'P96', was derived from selfed Roger's Red. It had a total of 151 progeny lines with 64 (42\%) of them showing a normal tendril distribution pattern and 87 (58\%) a mutant pattern (Table 2).

\section{Phenotypic evaluation of tendril distribution patterns}

Young seedling vines from germinated seeds were initially planted in 10 $\mathrm{cm}$ pots and then, when they reached to the stage with 4-5 leaves, transferred to 4-liter pots. Both crossed and selfed populations were planted about the same time in a greenhouse. The greenhouse had an average temperature of $26.7^{\circ} \mathrm{C}$ and $14 \mathrm{~h}$ of light. All vines received routine fertilizer application and watering.

When the first tendril appeared, the node was marked with a label so that the node position could be easily followed later in a vine. Detailed observation of presence or absence of tendril was taken for each node in each vine. As a vine grew, more labels were used to keep track of the relative positions of nodes in the vine. Data from 18 or more nodes, after the first node with the tendril was observed, were taken and the tendril distribution pattern was determined accordingly. The phenotypes were classified into two categories. One was the wild-type phenotypes with two sequential tendrils followed by a skip: XXOXXO, where $X$ represents presence of a tendril and $\mathrm{O}$ absence of a tendril. Conversely, the mutanttype phenotype represented tendril distribution patterns different from the wild-type, including XXOOX (a double skip), XXOOOX (a triple skip) or XXOOXOO (double-double skip). Although the disrupted mutant pattern was frequently observed in the proximal node, at the nodes 4-7, it was also observed at the distal nodes, such as at the nodes 10 and later.

\section{Tissue sampling and pooling}

Determination of the tendril segregation pattern of a vine was usually completed when the vine grew to 18 or more nodes. On the basis of the observed patterns of tendril distribution, we grouped the vines into mutant and wild-type categories for each population. Then several bulks of samples were taken for both mutant and normal vines for each population. Each bulk samples consisted of several individual vines from which the two youngest tendril closest to the first open leaf in the apex were taken (Table 1). To maximize the bulk contrast of wild-type and mutant phenotypes, we selected those vines that showed similar disrupted phyllotactic patterns as much as possible in the mutant bulk samples and those vines which had no broken shoots in the wild-type bulk samples. In the end, a total of $21 \mathrm{P} 96$ and 45 P65 progenies were pooled into 10 bulk samples for the construction of RNA-Seq libraries (Table 1). The bulk samples were frozen in liquid nitrogen and stored in $-80 \mathrm{C}$ freezer until further processed.

\section{RNA-Seq library construction and sequencing}

RNA-Seq libraries were constructed according to the protocols of Wang et $a l .{ }^{25}$ and Zhong et al. ${ }^{26}$ Briefly, $20 \mu \mathrm{g}$ total RNA was enriched for mRNA
Table 2. Chi-square significance tests of mutant versus wild-type segregations of tendril distribution patterns in fitting the gene action model of two epistatic supplementary dominant loci in selfed (P96) and cross (P65) populations, respectively

\begin{tabular}{|c|c|c|c|c|}
\hline \multirow[t]{2}{*}{ Phenotype } & \multicolumn{2}{|c|}{ P96 selfed population } & \multicolumn{2}{|c|}{ P65 cross population } \\
\hline & Observed & $\begin{array}{l}X^{2} \text { fit test for } \\
\text { fitting } 9 \text { mutant:7 } \\
\text { wild-type }\end{array}$ & Observed & $\begin{array}{l}X^{2} \text { fit test for } \\
\text { fitting } 1 \text { mutant: } \\
3 \text { wild-type }\end{array}$ \\
\hline Mutant & 87 & $0.73^{\mathrm{ns}}$ & 35 & $0.12^{\mathrm{ns}}$ \\
\hline Wild-type & 64 & & 140 & \\
\hline Total & 151 & & 175 & \\
\hline
\end{tabular}


using oligo (dT) magnetic beads and then fragmented by incubating at $94^{\circ} \mathrm{C}$ for $5 \mathrm{~min}$ in first strand synthesis buffer. From the fragmented mRNAs, first strand cDNA were synthesized with random hexamer-primer using Superscriptlll Reverse Transcriptase (Invitrogen, Carlsbad, CA, USA). The resulting CDNAs were purified with Agencourt RNAClean XP beads (Beckman Coulter Genomics, Danvers, MA, USA), followed by end repair and $d A$-tailing (NEB, Ipswich, MA, USA), and then ligated with Y-shaped adapters using a concentrated T4 DNA ligase (Enzymatics, Beverly, MA, USA). The adaptor-ligated cDNAs were size-selected with Ampure XP beads (Beckman Coulter Genomics, Danvers, MA, USA) before PCR amplification with indexed primers. The RNA-Seq libraries amplified with indexed primers were sequenced using an Illumina HiSeq system at the Biotechnology Resource Center of Cornell University (Ithaca, NY, USA).

Sequence data processing and differential expression analyses Raw RNA-Seq sequence data in fastq format were cleaned by removing sequence artifacts such as adapter sequences, low quality trailing and leading reads using FastQC ${ }^{27}$ and Trimmomatic (Illumina, San Diego, CA, USA). The cleaned sequence reads were then individually aligned to the Vitis reference genome $(12 \times V$. vinifera, Phytozome ver 12$)$ using the recommended alignment parameters for splice-aware transcriptome mapping as outlined in the Tophat2 workflow (http://ccb.jhu.edu/soft ware/tophat/manual.shtml). Intermediary processing of the mapped reads (sam and bam files) such as read deduplication and read group renaming was done using Samtools, ${ }^{28}$ Bamtools and Picardtools. ${ }^{29}$

Once aligned, the count data required by edge $R$ were obtained as a sum of overlapping reads on the annotated gene feature (gff3 file) using HTseq. ${ }^{30}$ Differential gene expression analysis was done in $\mathrm{R}$ using the Bioconductor package, edge-R. ${ }^{31}$ The standard normalization step that adjusts differences in initial library sizes was done using the default trimmed means of median (TMM) method. TMM is a normalization of library reads based on the scaling of the library size that minimize the logfold change. ${ }^{31}$ In addition, the ad-hoc minimum expression level for downstream analyses was set at 2 (count per millions of reads aligned) CPM for all libraries. DEGs were declared significant at the FDR $\leqslant 0.05$ and a 1.5 -fold change.

Functional and network co-expression analyses

To relate the biological significance of the discovered DEGs, gene ontology (GO) enrichment was conducted using Plant MetGenMAP. ${ }^{32}$ The resulting list of GO terms and their $P$ values were reduced to a representative GO terms by clustering similar terms and projected into an MDS plot, as implemented by a web-based GO enrichment engine, Revigo. ${ }^{33}$

Transcription factor gene families as well as gene annotations were identified by cross-referencing two online plant functional databases: Plant MetGenMAP ${ }^{32}$ and PlantTFD ver. $4 .{ }^{34}$ Regulatory cis network prediction and TF enrichment tests were facilitated using the algorithm implemented by Plant RegMap. ${ }^{34}$

To assess changes in co-expression topology in the mutant and wildtype background, network-based analysis was carried out using the differential dependency network (DDN) algorithm ${ }^{35}$ as implemented in its Cytoscape-based version, knowledge-fused differential dependency network (KDDN), ${ }^{36}$ where the predicted cis-regulatory connections among the DEGs determined from Plant RegMap, ${ }^{34}$ was used as a priori information. Cytoscape $^{37}$ was used to visualize the resulting co-expression network.

\section{RESULTS}

Phenotypic observation of tendril distribution

Among 151 self-pollinated progeny seedling vines from the P96 population, we observed 87 vines with the mutant pattern of tendril distribution and 64 with the wild-type pattern (the normal tendril distribution pattern) (Table 2). The observations fit the expected 9 mutants: 7 wild-types segregation ratio for two loci with supplementary dominant interaction $(P$ value $=0.73)$ as proposed by Cousins and Zhong. ${ }^{23}$ Similarly, among 175 progeny lines derived from the cross population P65, we observed 35 mutant and 140 wild-type vines, also fitting the expected segregation pattern of 1 mutant: 3 wild-type $(P$ value $=0.12)$ (Table 2).
Expression profile of DEGs

A total of 43.5 million 100 -bp single-end reads were generated in this study, and 30.5 million (70-80\%) were uniquely aligned to the reference genome $(12 \times V$. vinifera, Phytozome ver 12$)$. These uniquely matched transcripts were used for downstream differential expression analyses as implemented by edgeR. ${ }^{31}$ On the basis of a preliminary exploratory analysis of the data, we set a minimum threshold of at least 2 CPM in all the RNA-Seq sample libraries for each gene to reduce potential false positives. As a result, a total of 13375 expressed genes were retained. A multidimensional scale (MDS) analysis of these expressed genes revealed clear contrasts between mutant and wild phenotypes ( $y$-axis), and between different genetic backgrounds ( $x$-axis) (Supplementary Figure 1).

In the self-pollinated population P96, a total of 13940 expressed genes were detected, and 349 of them (2.6\%) were significantly differentially expressed. Among these 349 DEGs, 257 and 92 were up- and downregulated, respectively (Figure 1a). The upregulated DEGs had larger fold-change $(\sim 1 \times$ to $\sim 4 \times \log 2 \mathrm{FC})$ than the downregulated DEGs $(\sim 0.5 \times$ to $\sim 2 \times \log 2 \mathrm{FC})$. In the cross population P65, a total of 14238 expressed genes were detected, and 467 of them were differentially expressed (3.4\%). Among these 467 genes, 227 and 240 were up- and downregulated, respectively (Figure 1b). Similar to P96, the upregulated DEGs had larger fold-changes ranging from $\sim 1 \times$ to $\sim 4 \times \log 2 \mathrm{FC}$, whereas the downregulated DEGs were between $\sim 0.5 \times$ and $\sim 2 \times \log 2 \mathrm{FC}$. It was noted that increasing significance stringency from FDR $\leqslant 0.05$ to FDR $\leqslant 0.01$ retained about half of the DEGs in the self-pollinated population (177 from 349 genes) and about a third in the cross population (163 from 467). Most of the retained genes were of higher expression levels.

\section{Expression correlation of DEGs between P65 and P96}

A total of 13375 expressed genes were detected in both P65 and P96 populations. A moderate positive correlation $(r=0.46)$ between P65 and P96 was observed in the pairwise scatterplot of these genes based on their average fold-changes (Figure 1c). We further filtered the DEGs and focused our subsequent analysis on a subset of $324 \mathrm{DEGs}$, which had conforming expression profiles of at least 1.5 -fold change in both populations and were significant at FDR $\leqslant 0.05$ in at least one of the populations. This subset, composed of 201 upregulated and 123 downregulated genes, had a correlation coefficient $r=0.85$ of fold-changes of expression between the two populations.

\section{Uniquely expressed genes}

In the DEG analysis, we set a minimum expression level for a given gene at 2 CPM in all 10 libraries. Genes that were detected only in the wild or mutant libraries would be filtered out. We examined these excluded genes. We discovered four such genes that showed an average up- or downregulated expression level of $\sim 2$ CPM or more in a wild or mutant type with no reads detected in its counterpart.

Three genes were upregulated in the mutant background. The first one is a transcription factor (TF) bearing the AP2/ERF domain (GSVIVT01036388001) belonging to the integrase-type DNAbinding superfamily protein. This gene is homologous to Arabidopsis' ERF22, which encodes a DREB protein involved in embryogenesis. ${ }^{38}$ The other two upregulated genes were related to secondary metabolite synthesis, with one (GSVIVT01015165001, UDP-glycosyltransferase superfamily protein) found in the flavonol pathway, and the other (GSVIVT01009107001, PRX52) in the matairesinol biosynthesis belonging to the lignin biosynthesis pathway. The only downregulated gene, which had no aligned transcript in the mutant library, was annotated as laccase (GSVIVT01013693001), another gene in the lignin biosynthesis 

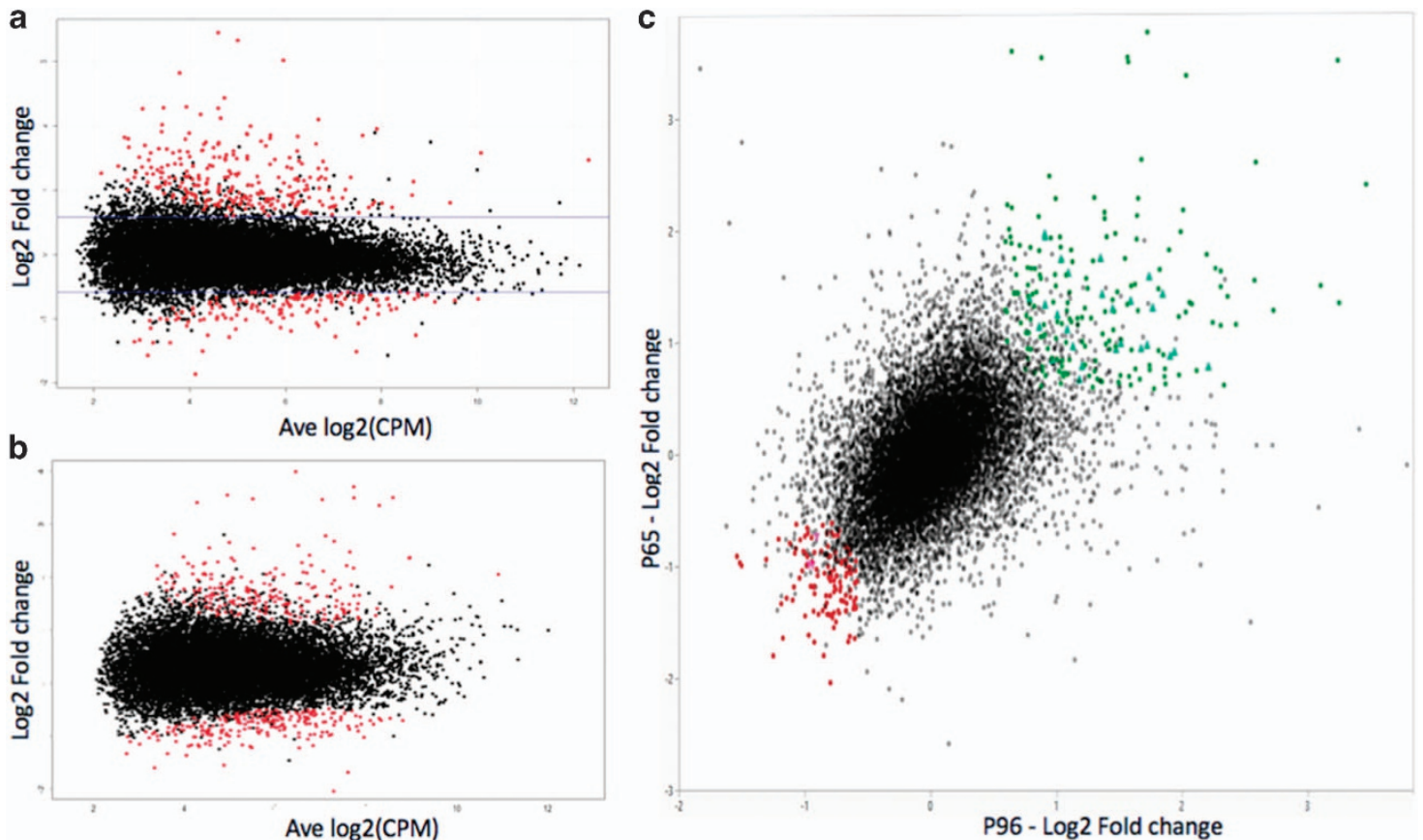

Figure 1. Expression profiles of differentially expressed genes (DEGs). (a) A log intensity ratio of expression versus average expression level (aka MA plot) of P96 depicting expression levels and fold-changes among 13940 expressed genes; 349 DEGs highlighted in red. (b) MA plot of P65 depicting expression levels and fold-changes among 14238 expressed genes; 467 DEGs highlighted in red. (c) Scatterplot of 13350 expressed genes between P65 and P96. Highlighted are the 324 DEGs, composed of 201 upregulated (green dots) and 123 downregulated (red dots) DEGs, which were used for subsequent analyses.

pathway. Lacasse is homologous to Arabidopsis' transparent testa 10 (TT10) involved in seed germination, root elongation and lignin degradation in the seed coat. $^{39}$

\section{Functional analyses of 324 DEGs}

Using Plant MetGenMap's gene ontology database, ${ }^{32} 191$ of the 324 DEGs (58\%) were matched with GO annotations. The larger GO biological processes among the DEGs were 'Cellular process', 'Response to stress', 'Metabolic process', 'Transport', 'Transcription' and 'Response to stimuli'. Subsequent GO enrichment analyses revealed that at a conservative cut-off threshold (FDR $<0.10), 19$ biological processes were enriched. As determined by using Revigo, ${ }^{33}$ these 19 biological processes grouped into four semantically similar biological processes: 'Response to biotic and abiotic stimuli', 'Plant signaling', 'Plant transport' and 'Transcriptional regulation and cell communication' (Table 3; Supplementary Figure 2). Although there was a large overlap among the member genes in these four clusters, each cluster was semantically distinct, defined by the frequency and identity of its member genes.

Cluster I roughly describes response to stimuli processes, having GO terms 'Response to chitin', 'Response to carbohydrate' and several stimuli-induced plant responses (Table 3). This cluster is comprised of 97 DEGs, majority of which were highly expressed and stress-inducible (Supplementary Table 1). Asparagine synthase (GSVIVT01024713001), a key gene in nitrogen assimilation and translocation ${ }^{40}$ and a stress-response gene in microbial infections like Xanthomonas, ${ }^{41}$ was the most upregulated gene among the $324 \mathrm{DEGs}$, with an average of fold of change at $3.4 \log 2 \mathrm{FC}$, and about twice the average positive fold-change of cluster I. On the other hand, cyclin B1-2 (GSVIVT01032782001), a gene that encodes a kinase-activating protein important to mitosis and cell cycle-related growth responses ${ }^{42}$ and triggered by many stress conditions such as high salinity, ${ }_{1}^{43}$ was one of the most downregulated gene with the fold of change at an average of $1.4 \log 2$ FC. Majority of the DEGs
Table 3. Significantly enriched $\mathrm{GO}$ biological terms revealed from 324 DEGs (FDR < 0.10) and the GO term cluster assignments

Significant GO term
Response to carbohydrate stimulus
Response to chitin
Response to organic substance
Regulation of response to stimulus
Response to endogenous stimulus
Response to chemical stimulus
Response to abiotic stimulus
Response to stress
Response to biotic stimulus
Response to ethylene stimulus
Jasmonic acid-mediated signaling pathway
Negative regulation of response to stimulus
Ethylene-mediated signaling pathway
Induced systemic resistance
Two-component signal transduction system (phosphorelay)
Amide transport
Urea transport
Regulation of cell communication
Regulation of gene expression

Cluster

in this cluster, especially those with large expression changes such as cytochrome P450 (GSVIVT01008261001), lysine-specific demethylase 3B (GSVIVT01026208001) and defensin protein (GSVIVT01010274001), were from different metabolic pathways but were directly or indirectly activated by biotic and abiotic stresses. ${ }^{44-46}$ Cluster I also includes 27 of the 30 differentially expressed TFs, 15 of which belong to the known major stressresponse TFs gene families AP2/ERF, NAC and WRKY. More interestingly, closer scrutiny of their binding sites and targets, as determined using Plant RegMap, ${ }^{34}$ revealed that about half of the DEGs (37 out of 70) in this cluster were the direct down- 
stream targets of these TFs. Included in the prominently upregulated targets ( $>1.7$ log2 FC) were dehydrin gene 1 (GSVIVT01018878001), a target of AP2/ERF; peroxidase gene (GSVIVT01009106001), a target of bHLH and TCP; and protein TIFY 5A (GSVIVT01021514001), a target of NAC. Among the prominently downregulated ( $>1.2 \log 2 \mathrm{FC}$ ) targets were syntaxin gene (GSVIVT01035559001), a target of WRKY; thiamine thiazole synthase (GSVIVT01012636001), a target of AP2/ERF; and lysinespecific demethylase 3B (GSVIVT01026208001), a target of NAC. The aforementioned target genes were known for their role in stress conditions. ${ }^{47-49}$ Thus, taken together, cluster I, which corresponded to 'Response to stimuli', was mainly comprised of biotic and abiotic stress-inducible genes and their transcriptional regulators.

Cluster II is composed of four symantically similar GO terms related to 'Plant signaling' (Table 3; Supplementary Figure 2). It is composed of 19 DEGs, 15 of which were TFs, whereas three of the remaining four DEGs were downstream targets to WRKY and TCP gene families (Supplementary Table 1). The WRKY-targeted gene families include the downregulated plant signaling gene syntaxin $^{50}$ (GSVIVT01035559001) and ABC transporter genes ${ }^{51}$ (GSVIVT01016999001), whereas the TCP-targeted genes included respiratory burst oxidase homolog (GSVIVT01019429001), a member of the redox-sensitive signaling. ${ }^{52,53}$ Cluster III corresponds to 'Transport' among the enriched biological processes, with two aquaporin homologs (GSVIVT01025038001, GSVIVT01016615001 ) that primarily mediate plant water transport activated during drought-stress conditions and ripening ${ }^{54,55}$ (Supplementary Table 1).

Cluster IV is a subset of 41 DEGs related to 'Transcriptional regulation and cell communication' processes (Table 3; Supplementary Figure 2). The upregulated genes in this cluster were mostly involved in stress-response mechanisms such as the cationic amino acid transporter (GSVIVT01034656001), a gene belonging to choline transporters activated during root-knot nematode infection, ${ }^{56}$ as well as MYC 2 (GSVIVT01013156001), a bHLH domain-carrying member of MYC-related gene family whose many biological roles include interaction with Jasmonatezim domain (JAZ) to elicit drought tolerance in plants. ${ }^{57}$ On the contrary, the downregulated genes seemed to reflect more developmental regulatory genes. For example, the pseudo-response regulator gene (GSVIVT01032644001) was associated with circadian clock regulation, as well as phytochrome-dependent transduction, ${ }^{58}$ whereas genes such as RNA-binding gene (GSVIVT01009045001), neuroguidin gene (GSVIVT01009200001) and endoribonuclease dicer-like protein encoding gene (GSVIVT01027460001) were genes broadly related to post-transcriptional gene regulation. ${ }^{59}$ Similar to the previous clusters, cluster IV includes TFs and their downstream targets (Supplementary Table 1). Notable upregulated target genes among the $26 \mathrm{TFs}$ in cluster IV were protein TIFY 5A (GSVIVT01021514001, a NAC downstream target), GID1, an important GA receptor gene (GSVIVT01011037001; a AP2/ERF downstream target) and putative-ubiquitin conjugating enzyme (GSVIVT01034196001, a TCP downstream target). Protein TIFY is a Jasmonate-ZIM domain protein 8 homolog belonging to the JAZ protein family and a noted key gene in reproductive developmental processes. ${ }^{49}$ GID1 is part of the GA pathway, a key component GA:GID1 complex bound by DELLA in maintaining the critical GA homeostasis. ${ }^{60}$ On the other hand, among the highly downregulated targets was lysis-specific demethylase 3B gene (GSVIVT01026208001; a NAC target). Its homolog in Arabidopsis, IBM1, was reported to mediate histone methylation processes involved in arrested flower and pollen development. ${ }^{61}$ Another important downregulated gene in this cluster is MYB12, a flavonoid biosynthesis activator. ${ }^{62}$ In grapevine, the secondary metabolites, such as flavonols, flavonoids and anthocyanins, have major roles in plant defense response ${ }^{63}$ in addition to their roles in wine-related quality traits. ${ }^{64}$ Apparently, this cluster of 'Transcriptional regulation and cell communication' biological processes includes the genes controlling developmental plant hormones, mainly GA and ABA, as well as secondary metabolites such as flavonoids, and even methylation-mediated regulations that involves dicer and RNA-binding protein. A TCPdomain TF, TCP3, was only found in this cluster but not in the others, suggesting that the molecular mechanism attributed to TCPs_-plant form and structure-might be a significant part that defines an enriched transcription regulation and cell communication processes found in tendril phyllotaxy.

Unfortunately, only about $60 \%$ of the 324 DEGs could be accounted for in these GO enrichment analyses. Nevertheless, the analyses revealed that tendril/shoot phyllotaxy was intricately connected with the plant's regulatory control for responses to internal and external stimuli, a large part of which might be, as shown in the enriched gene sets, mediated through cis-regulation.

\section{Dependency network analysis}

To examine possible transcriptome-wide rewiring among the 324 DEGs, we carried out a network-based expression analysis using DDN. DDN examines changes in co-expression topology between two conditions (in this case, mutant and wild-type phenotypes) using a network-learning algorithm, to detect selectively activated or deactivated regulatory mechanisms. We used an enhanced version, $\mathrm{KDDN}^{36} \mathrm{KDDN}$ allows users to incorporate established biological information such as the pairwise cis-regulatory connections obtained from Plant RegMap ${ }^{34}$ as a priori knowledge in dependency network construction.

To examine the most plausible and relevant transcriptome network rewiring associated with tendril phyllotaxy from the complex KDDN-generated dependency network, we extracted the ten most connected (hub) genes and the first-degree connections around them. The extracted subnetwork consists of a total of 96 DE genes (Supplementary Figure 3). Hub genes are points of interest in expression networks because they, and connections around them, are likely to correspond to relevant biological regulatory roles in the proposed network. ${ }^{65}$

Table 4 listed the hub genes identified from the dependency network analysis of the 324 DEGs. Most of these hub genes have known regulatory roles in plant stress responses. For example, two of the hub genes in Table 4 were related to the AP2/ERF gene family, which mediates developmental processes such as flower development, ${ }^{66}$ as well abiotic stress responses such as drought, high salinity and extreme temperatures. ${ }^{55}$ The first listed AP2/ERF hub gene, AtWind1, is a noted TF mediating callus formation during wound injury. ${ }^{67}$ It has 10 connections in the network, including genes in the signaling cascade pathway MAPK and calmodulin-binding protein kinase (Table 4; Figure 2a). As further revealed by the KDDN-generated dependency network, AtWind1 had a significantly strong connection (FDR $<0.01)$ in the mutant background with PLATZ TF (Figure 2a), another plant-specific TF involved in cell differentiation. ${ }^{68}$ The other listed AP2/ERF hub gene is ERF17 (Figure 2a), a homolog of Arabidopsis' AtERF17. The dependency network suggested that the ERF17 had connections with genes having wide biological roles such as transport (hexose transporter, GSVIVT01017937001; plant lipid transfer proteins, GSVIVT01015895001), hormone balance (IAA-amino acid hydrolase, GSVIVT01008852001) and reactive oxygen species (ROS)mediated defense response (Roxy19, GSVIVT01021124001). Interestingly, the signal transducer Roxy19 belongs to glutaredoxin (GRX) gene family, a group downstream to TCP gene family regulation, ${ }^{69}$ which has been found related to shoot phyllotaxy in maize ${ }^{70}$ and petal formation in Arabidopsis. ${ }^{71}$ In addition, ERF17 belongs to the subclade of AP2/ERF gene family that readily interacts with GRAS TFs. ${ }^{72}$ 
Table 4. Hub genes identified in the KDDN-generated dependency network

\begin{tabular}{lccll}
\hline Gene ID & Average log2 FC & No of connection & Arabidopsis annotation & Biological pathway/TF family \\
\hline GSVIVT01009007001 & 1.22 & 10 & AtWind1 & AP2/ERF TF \\
GSVIVT01015037001 & 1.64 & 9 & AtERF17 & AP2/ERF TF \\
GSVIVT01030219001 & 1.45 & 7 & PRX52 & Lignin biosynthesis \\
GSVIVT01033485001 & -1.20 & 11 & Une10 & bHLH TF \\
GSVIVT01019515001 & 1.72 & 7 & Unknown protein & - \\
GSVIVT01031462001 & 1.22 & 9 & Superoxide dismutase & Ethylene biosynthesis \\
GSVIVT01027027001 & 2.13 & 7 & Acidic endochitinase & Chitin degradation \\
GSVIVT01022164001 & -1.25 & 9 & D-alanyl-D-alanine carboxypeptidase, putative & Protein transport \\
GSVIVT01021507001 & 1.37 & 10 & 9-cis-epoxycarotenoid dioxygenase 2 & ABA pathway \\
GSVIVT01009045001 & -1.03 & 8 & RNA-binding protein & Post-transcriptional regulation
\end{tabular}

Another hub gene listed in Table 4 is an RNA-binding homolog, one of the genes in the subset of enriched GO biological process 'Plant regulation and cell communication'. Although its molecular function has yet to be fully elucidated, this RNA-binding gene is believed to primarily have a role in post-transcriptional gene regulation. ${ }^{73}$ Our KDDN network analysis suggested a significant connection in the mutant background with a calcium kinase 1-related gene (GSVIVT01022606001), a gene that phosphorylates phenylalanine ammonia lyase (PAL) and serves as a key enzyme in pathogen defense ${ }^{74}$ (Supplementary Figure 3). In addition, the RNA-binding hub gene's connection was deactivated to a putative regulatory connections of NAC002 (GSVIVT01008839001), a TF active in abiotic stresses and pathogen infection response ${ }^{75}$ (Supplementary Figure 3). Taken together, the proposed dependency network emanating from the RNA-binding gene in this study re-confirmed the possible role of post-transcriptional regulation in plant stress response ${ }^{76}$ and/or shoot architecture. ${ }^{77}$

Perhaps the more interesting hub gene is the peroxidase gene (GSVIVT01030219001). Its Arabidopsis homolog, PRX52, is a major lignin and secondary cell wall biosynthesis gene especially in the stem and xylem vessels; loss-of-function mutants for this gene in Arabidopsis showed $70-80 \%$ reduction in lignin content, especially the syringyl lignin. ${ }^{78}$ It was proposed that it is one of the points of regulatory control in $\mathrm{ABA}$-mediated defense responses during bacterial, fungal and insect attack. ${ }^{47}$ In our proposed KDDN network where it had seven connections (Table 4), PRX52 had a significant connection $\left(P_{\text {val }}<0.01\right)$ in the mutant background with a glucosinate transporter 2 (GSVIVT01008072001), a member of the nitrate transporter1/peptide transporter family (NPF) transporters (Figure 2b). NPF were recently reported as critical carriers of GA and ABA hormone in grapevine. ${ }^{79,80}$ In addition, PRX52's connection was deactivated in the mutant background with a carboxykinase gene (GSVIVT01005596001), which encodes a GTP protein in the gluconeogenesis pathway, which in turn is central to G-protein-mediated signal transduction in plant immunity. ${ }^{81}$ Interestingly, although the KDDN connections were not significantly rewired, PRX52 was connected to two downregulated TFs that have key regulatory roles in plant form and defense responses (Figure $2 \mathrm{~b}$ ). Our dependency network correctly depicted PRX52 as one of the downstream targets of TCP3, a class II CIN-TCP TF. The class II TCP transcription factor that bears the CINCINATA motif such as TCP $3^{69}$ has been shown to regulate the morphogenesis of shoot lateral organs, as well as correct petal and stamen development ${ }^{82,83}$ and defense responses. ${ }^{84}$ It is highly possible that PRX52's role in lignification might be related to TCP3's wider role in plant form and stress response. PRX52 was also connected to a flavonol-specific activator, MYB12. ${ }^{85}$ The flavonol and flavonoid pathways produce secondary metabolites and share the phenylpropanoid pathway with lignin biosynthesis, pathways that have been implicated in plant defense responses in grapevine. ${ }^{86}$
Regulatory connection among TFs and their target genes

The considerable proportion of TFs and their targets among the sets of enriched biological processes earlier examined suggested a substantial role of cis-regulation in the discovered DEGs. Indeed, based on the Plant RegMap's cis-regulatory database, ${ }^{34} 21$ TFs (out of the 30) and their predicted targets accounted for about $35 \%$ of the observed DEGs (123 of the 324 DEGs). A formal enrichment test revealed that nine genes belonging to five TF families had significantly over-represented targets: AP2/ERF, WRKY, NAC, bHLH and TCP (Table 5). As was earlier noted, the AP2/ERF domain family has the highest number of downstream targets, with three AP2/ERF genes accounting for $\sim 28 \%$ of the total DEGs (Table 5). With 40 targets, ERF17 (GSVIVT01015037001) dominated the possible cis-binding interactions in the observed DEGs, perhaps due to its cross interactions with other TFs. ERF17 belongs to the A-5 DREB subfamily that interacts with other DREB subfamilies in regulating cold and high-salinity tolerance. ${ }^{55}$ In addition to its putative role in organic acid accumulation, ${ }^{87}$ ERF17 is also classified to belong to the gibberellin-related clade of the AP2/ ERFs family because of its tendency to interact readily with DELLA of the gibberellin pathway. ${ }^{72}$ With 23 downstream targets, the second ranking gene belonging to the AP2/ERF gene family is WIND1 (GSVIVT01009007001), a noted TF in cell differentiation. ${ }^{67}$ The third AP2/ERF gene (GSVIVT01021098001) is a homolog of ERF09, an AP2/ERF known for its role in pathogen-related defense responses. ${ }^{66}$ It is noteworthy to recognize that both ERF17 and WIND1 were also the most connected hub genes in the expression topology-driven dependency network (Table 4; Supplementary Figure 3), reinforcing the substantial role of cis-regulation among co-expression profiles of the 324 DEGs.

A bHLH homeodomain TF (GSVIVT01018165001) was also significantly enriched with downstream targets of 20 DEGs (Table 5). This particular bHLH TF is homologous to UNE10, a regulator active during seed fertilization in Arabidopsis. ${ }^{88}$ The bHLH gene family are also one of the key components in the ternary complex of TFs (MYB-bHLH-WD40) required for the initiation of the anthocyanins and proanthocyanidins. ${ }^{89}$ Interestingly, it was also found to be one of the major hub genes in the dependency network as revealed in this study (Table 4; Supplementary Figure 3).

NACs are largely involved in the ABA-dependent stress signaling pathway. Two NAC TFs were found significantly enriched in this study. The first one was homologous to AtAF1, which in Arabidopsis was mediated via the ROS signal transduction pathway in responding to many abiotic stresses and pathogen infections stimuli. ${ }^{75}$ The second NAC gene (GSVIVT01014403001) was a homolog of RD26, which was noted to be insensitive to jasmonic acid-related stress signaling. ${ }^{90}$

TCP3 was also among the enriched with a predicted downstream target of 24 DEGs. A class II CIN-TCP, TCP3 is recognized a key regulatory control of shoot morphogenesis through negative 
a
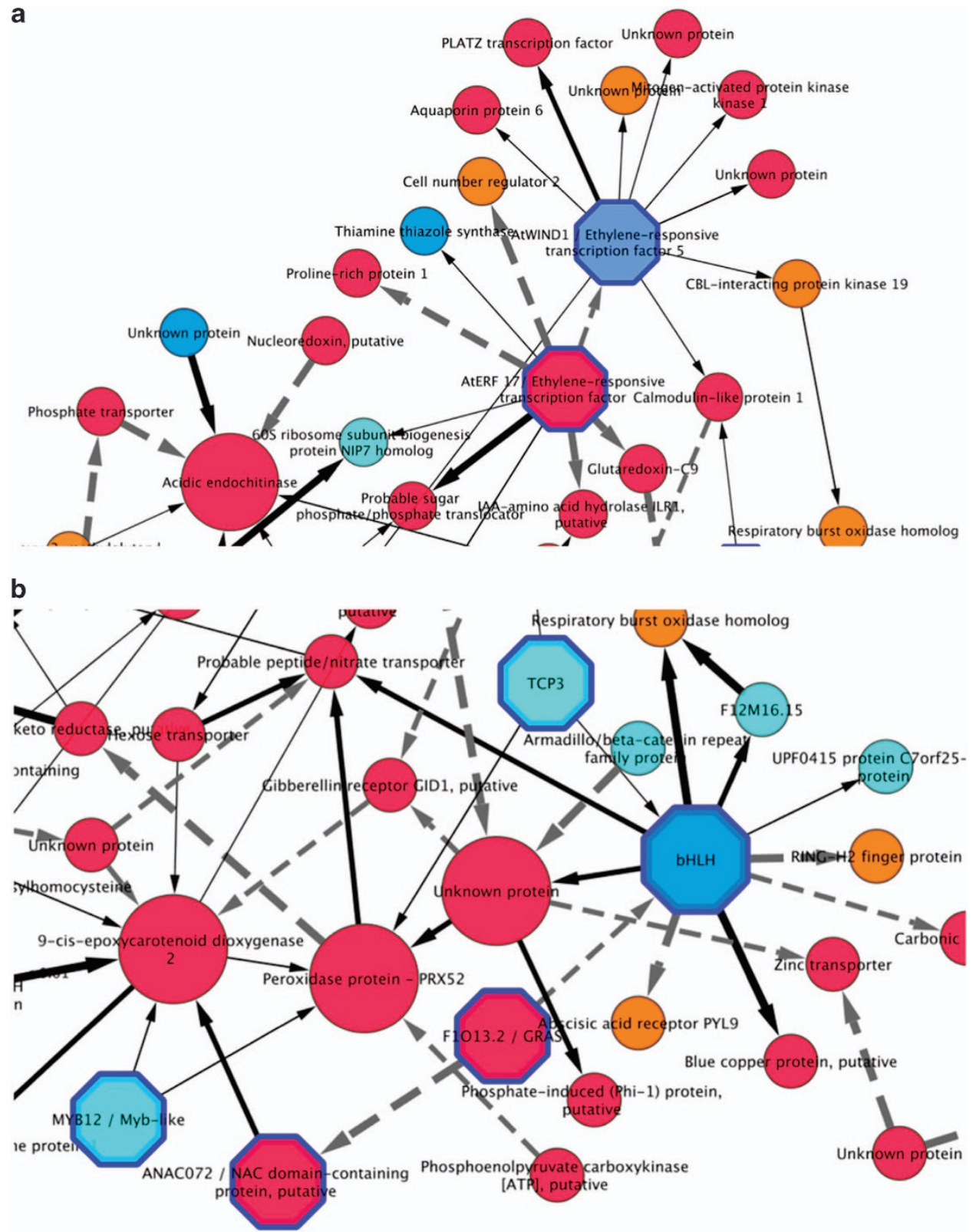

Figure 2. (a) AtERF17 and AtWIND1 (AP2/ERF) hub genes and their immediate connections in KDDN-generated dependency network. Orange and red nodes are moderately to highly upregulated genes. Light blue to dark blue nodes are moderately to highly downregulated genes. Octagon-shaped nodes are transcription factors. Connecting lines represent directional co-dependency in expression, with thicker lines indicate highly significant $\left(P_{\text {val }}<0.01\right)$ connections in the mutant background, whereas broken lines indicate significantly deactivated connections in the mutant background. The size of a node is proportional to the number of connecting lines involved. (b) TCP3 and MYB12 relevant connections in the KDDN-generated dependency network among the 10 most connected hub genes. Orange and red nodes are moderately to highly upregulated genes. Light blue to dark blue nodes are moderately to highly downregulated genes. Octagon-shaped nodes are transcription factors. Connecting lines represent directional co-dependency in expression, with thicker lines indicate highly significant $\left(P_{\text {val }}<0.01\right)$ connections in the mutant background, whereas broken lines indicate significantly deactivated connections in the mutant background. The size of a node is proportional to the number of connecting lines involved.

regulation of the boundary-specific genes through miRNA induction. $^{83}$

Tendril and flower-identity genes

In many ways, the complex regulatory dynamics and the relatively large number of development-associated TFs uncovered in this study are typical of actively developing organs such as a tendril. The grapevine tendrils, however, are homologous to inflorescences and were reported to express flower-identity genes such as AP1, FUL, FT and LFY.,13,15 We observed that all the homologs of the floral-identity genes AP1, FUL, FT were expressed at varying expression levels in our experiment. However, they were not differentially expressed between mutant and wild-types. We also confirmed that the LFY homolog was not expressed in the tendril transcriptome, which was in agreement with the observation that the gene seemed expressed only in grapevine inflorescence and not in tendril. ${ }^{7,13}$ 
Table 5. Most significantly enriched transcription factors among the 324 DEGs identified in this study

\begin{tabular}{lcclll}
\hline Gene ID & Total DEGs & No. of targets & FDR & Annotation (Arabidopsis best hit) & TF family \\
\hline GSVIVT01015037001 & 324 & 44 & $5.06 \mathrm{E}-08$ & AtERF17 & AP2/ERF \\
GSVIVT01018165001 & 324 & 20 & $1.14 \mathrm{E}-04$ & Une 10 & bHLH \\
GSVIVT01009007001 & 324 & 23 & $4.53 \mathrm{E}-04$ & AtWIND1 & AP2/ERF \\
GSVIVT01014236001 & 324 & 27 & $9.79 \mathrm{E}-04$ & TCP3 & TCP \\
GSVIVT01012682001 & 324 & 10 & $4.33 \mathrm{E}-03$ & WRKY6 & WRKY \\
GSVIVT01027069001 & 324 & 10 & $7.15 \mathrm{E}-03$ & WRKY30 & WRKY \\
GSVIVT01021098001 & 324 & 23 & $7.94 \mathrm{E}-02$ & ERF9 & AP2/ERF \\
GSVIVT01008839001 & 324 & 7 & $8.69 \mathrm{E}-02$ & AtAF1 & NAC \\
GSVIVT01014403001 & 324 & 5 & $1.05 \mathrm{E}-01$ & RD26 & NAC \\
\hline
\end{tabular}

\section{DISCUSSION}

Unique to grapevine shoot growth is the simultaneous differentiation of reproductive and vegetative organs in the same meristematic cells in shoot apex, and a number of studies have contributed to our understanding of the underlying biology of this phenomenon. ${ }^{4,91,92}$ Although the growth pattern of vine shoot development remains largely a point of conjecture as sympodial or monopodial growth, ${ }^{18,93}$ the resulting shoot phyllotaxy has been found more or less constant within given taxonomic groups and can be used to differentiate them in Vitaceae. ${ }^{18}$ The shoot phyllotaxy in vines is presumably under genetic control, but experimental evidence to support the claim is scarce. Recently, Cousins et al. ${ }^{22}$ reported that seedling populations derived from self-pollination of interspecific Vitis hybrid cultivars and from hybridization of Vitis hybrid cultivars, showed a high incidence of abnormal or mutant tendril distribution; and a subsequent study of the tendril segregation patterns from the self-pollinated and crossed progeny derived from the hybrid suggested that the mutant type was likely controlled by two epistatic loci ${ }^{23}$ with a supplementary dominant-gene action. In this study, we observed similar segregation patterns from the same sets of populations reported by Cousins and Zhong ${ }^{23}$ and confirmed that the hypothesis they proposed offered the most satisfactory explanation of the genetic control of the tendril phyllotaxy or distribution patterns in the Vitis species. Further validation of the hypothesis can be carried out, for example, by mapping QTL loci controlling the tendril distribution patterns using appropriate mapping populations such as those used in the present study.

To elucidate the genetic and molecular processes involved in tendril phyllotaxy, we carried out a RNA-Seq differential expression analysis of bulked wild-type and mutant samples of both selfpollinated and cross populations in this study. As is the case for all profiling studies, data quality is critical for drawing valid conclusions. To reduce potential false positives, we used a minimum expression threshold of at least 2 CPM for all the genes across all the RNA-Seq sample libraries in this study. The quality of the resulting data was satisfactory as clear pattern contrasts between the wild and mutant phenotypes were revealed by a MDS analysis of the expressed genes.

In this study, we found 324 DEGs, with at least 1.5-fold changes for each individual gene in the mutant and wild-type bulk samples from both self-pollinated and outcross genetic background involving the Vitis hybrid 'Roger's Red'. The high correlation coefficient $(r=0.85)$ of the fold-changes of expression of these genes between the two populations suggested that these 324 genes were likely involved in the mutant tendril phenotype in the populations studied. When we examined the subsets of DEGs within the enriched GO biological processes, a substantial proportion of them were TFs and their predicted target genes. This highly suggested a substantial role these TFs may have in the observed differential expression. Indeed, consistent with early reports, ${ }^{15}$ the expressed genes in grapevine tendril included the
AP2/ERF, NAC and WRKY TF families. These TF gene families were known to mediate plant defense response, ${ }^{94}$ hormone response ${ }^{66}$ and abiotic stress tolerance, ${ }^{95}$ and hence it was not a surprise that they had a large role in the resulting enriched GO terms. Molecular studies of tendrils in other species have shown that TFs likely had a significant role in tendril development. For example, the leafletderived tendrils of garden pea (Pisum sativum) was attributed to loss-of-function of the $\mathrm{Tl}$ gene, which encodes a Class I homeodomain leucine zipper TF. ${ }^{96}$ Similarly, the tendril-less melon (Cucumis melo) mutant, Chiba Tendril-Less (ctl), was thought to be a single-base deletion in a CmTCP1 gene (a TCP TF gene). ${ }^{97}$

Our DDN analysis, which examined the transcriptional 'rewirings' between the mutant and normal phenotypes, provided detailed information of how various TFs might contributed to the tendril development. The condition-specific (mutant versus wild) connections among the top 10 hub genes revealed diverse and complex overlaps of several regulatory and metabolic pathway genes, which mainly were related to stress responses, plant hormones and secondary metabolites. The emerging theme from the expression and network enrichment analyses was the apparent importance of cis-regulatory interactions of the major stressresponsive TFs in tendril phyllotaxy. This was reflected in the fact that the combined number of downstream targets of the TFs AP2/ERF, WRKY, NAC and TCP accounted for about 38\% (123 of the 324) of the total discovered DEGs. In addition, a dependency network constructed with these cis-regulatory connections as $a$ priori information revealed that the extensively connected hub genes were either TFs or important genes in developmental pathways. More importantly, the significant re-wirings of these hub genes involved at least one (de)activated TF.

MYB12 is a flavonol-specific TF named VVMYBF1 in grapevine. ${ }^{62}$ It regulates the first step in flavonol biosynthesis (that is, Flavonol Synthase 1 or FLS) and thus is a key activator of flavonol biosynthesis gene. ${ }^{89}$ In addition to the widely recognized role in controlling the synthesis of flavonols, VVMYBF1 also forms a ternary complex with the TFs bHLH and WD40, referred as the MBW ternary complex, to activate the anthocyanin synthesis pathway. ${ }^{89}$ As anthocyanins and flavonoids are important domestication traits in grapevine related to berry and wine-making quality, the roles of VVMYBF1 and a host of other MYB TFs and regulators in this pathway have been widely expanded to include not only pigmentation, berry ripening and cell fate, but also plant defense response, drought tolerance, pathogen resistance and lightsensing response. ${ }^{98}$ Interestingly, it has recently been shown that TCP3 enhanced flavonoid biosynthesis by interacting with MYB12 and thus (de)stabilizing a MBW complex. ${ }^{89}$ It was reported that a destabilized flavonol and anthocyanin pathway by TCP3 interaction affected the auxin biosynthesis and auxin-related processes. ${ }^{99,100}$ Furthermore, in Arabidopsis, such TCP-MYB12 interaction was shown to affect several pathways that manifested into an altered leaf phyllotaxy, abnormal vasculature patterning, reduced apical dominance, impaired root development and reduced organ size. ${ }^{101}$ 
Another TF that may significantly affect tendril phyllotaxy is ERF17. ERF17 is an AP2/ERF TF with the single-most number of DEG targets ( $13 \%$ of 324 DEGs). Our co-dependency network analysis suggested that ERF17 had a significant interaction with ROXY19, which belongs to the glutaredoxin (GRX) gene family, a class of oxidative response genes. In Arabidopsis, two GRX genes were shown to have important roles in petal formation via posttranslational modification. ${ }^{71}$ This finding offers an interesting avenue for further investigation, as grapevine tendril is a modified inflorescence ${ }^{7,13}$ expressing flower development genes. ${ }^{15}$ Interestingly, one of the four genes identified to be uniquely expressed in the mutant background was also an AP2/ERF TF (ERF22, GSVIVT01036388001).

In addition to the TFs discussed above, we have also found some lignin-related genes, which were likely involved in the mutant phyllotaxy observed. Among the four uniquely expressed genes we examined (only expressed in the wild-type or mutant background), we found two lignin-related genes, PRX52 (GSVIVT01009107001) and lacasse (GSVIVT01013693001), whose expressions were, respectively, enhanced and suppressed in the mutant background. PRX52 was previously found to have a key role in lignin synthesis, ${ }^{78}$ whereas the lacasse gene was involved in lignin degradation. ${ }^{39}$ Interestingly, our dependency network analysis independently showed that the same lignin biosynthesis gene, $P R X 52$, was co-regulated by two important transcription factors, TCP3 and MYB12, which were identified to have key roles in tendril phyllotaxy, as discussed earlier. These evidences collectively suggested that the genes in the lignin pathway might have contributed to the tendril phyllotaxy of the mutant observed in this study. Indeed, lignification, cell wall development and cell proliferation are processes ontologically related with shoot architecture development ${ }^{102}$ and likely with tendril development as well.

To conclude, tendril phyllotaxy is an important developmental trait and likely has a complex basis of genetic control. Unfortunately, we have very little knowledge about the global molecular processes of the development of the trait. Our work in this study is the first attempt to fill this knowledge gap in the literature. Through analyzing 324 DEGs from both selfed and outcrossed populations, we found several TFs, which likely had significant roles, through regulating DEGs and others, in contributing to the development of tendril phyllotaxy. TCP3, a known master integrator in growth and development, appeared to be one of the key TF genes involved in the process. Among the structural genes, we have found several lignin-related genes likely involved in tendril development. Like in many profiling studies, the results reported in this study would have to be validated in the future experiments. Nevertheless, these results should provide the first insight of the complex molecular events involved in grapevine tendril development.

\section{CONFLICT OF INTEREST}

The authors declare no conflict of interest.

\section{ACKNOWLEDGEMENTS}

We wish to thank Debra Johnston, Della Cobb-Smith, Michael Brown and Venna Wang of USDA-ARS Grape Genetics Research Unit for providing their assistance in maintaining the vine populations and collecting phenotypic data in the greenhouses, and Bill Srmack, John Keeton and Bob Martens of Horticulture Section, School of Integrative Plant Sciences, Cornell University and USDA-ARS, Plant Genetic Resources Unit for their assistance in maintaining the vines in field. JA and JC are participants of the ORISE-ORAU Education and Training Program.

\section{REFERENCES}

1 Carmona MJ, Chaïb J, Martínez-Zapater JM, Thomas MR. A molecular genetic perspective of reproductive development in grapevine. J Exp Bot 2008; 59: 2579-2596.

2 This P, Zapater JM, Péros J-P, Lacombe T. Natural variation in Vitis. In: AdamBlondon AF, Martínez-Zapater JM, Kole C (eds). Genetics, Genomics and Breeding of Grapes. Science Publishers: New Hampshire, 2011, pp 30-67.

3 Carmona M, Greven M, Winefield CS, Trought MCT, Raw V. The flowering process of Vitis vinifera: a review. Am J Enol Vitic 2009; 60: 411-434.

4 Gerrath J, Posluszny U, Melville L. Taming the Wild Grape: Botany and Horticulture in the Vitaceae. Springer International Publishing: Switzerland, 2015, pp 1-194.

5 Keller M. Botany and anatomy. In: The Science of Grapevines. Elsevier Inc: San Diego, CA, 2015, pp 1-47.

6 Srinivasan C, Mullins MG. Control of flowering in the grapevine (Vitis vinifera L.). Plant Physiol 1978; 61: 127-130.

7 Boss PK, Buckeridge E, Poole A, Thomas MR. Review: new insights into grapevine flowering. Funct Plant Biol 2003; 30: 593-606.

8 Fasoli M, Dal Santo S, Zenoni S et al. The grapevine expression atlas reveals a deep transcriptome shift driving the entire plant into a maturation program. Plant Cell 2012; 24: 3489-3505.

9 Wen J. Vitaceae. In: Kubitzki K (ed). The Families and Genera of Vascular Plants. Springer: Berlin, 2007, pp 466-478.

10 Pratt C. Reproductive anatomy in cultivated grapes-a review. Am J Enol Vitic 1971; 22: 92.

11 Posluszny U, Gerrath JM. The vegetative and floral development of the hybrid grape cultivar 'Ventura'. Botany 1986; 64: 1620-1631.

12 Gerrath JMM, Posluszny U, Dengler NGG. Primary vascular patterns in the Vitaceae. Int J Plant Sci 2001; 162: 729-745.

13 Zhang N, Wen J, Zimmer EA. Expression patterns of AP1, FUL, FT and LEAFY orthologs in Vitaceae support the homology of tendrils and inflorescences throughout the grape family. J Syst Evol 2015; 53: 469-476.

14 Isnard S, Silk WK. Moving with climbing plants from Charles Darwin's time into the 21st century. Am J Bot 2009; 96: 1205-1221.

15 Diaz-Riquelme J, Martinez-Zapater JM, Carmona MJ. Transcriptional analysis of tendril and inflorescence development in grapevine (Vitis vinifera L.). PLOS ONE 2014; 9: e92339.

16 Calonje M, Cubas P, Martínez-Zapater JM, Carmona MJ. Floral meristem identity genes are expressed during tendril development in grapevine. Plant Physiol 2004; 135: 1491-1501.

17 Carmona MJ, Cubas P, Martinez-Zapater JM, Martı M. VFL, the grapevine FLORICAULA/LEAFY ortholog, is expressed in meristematic regions independently of their fate. Plant Physiol 2002; 130: 68-77.

18 Gerrath JM, Posluszny U. Shoot architecture in the Vitaceae. Can J Bot 2007; 85: 691-700.

19 Louarn G, Guedon Y, Lecoeur J, Lebon E. Quantitative analysis of the phenotypic variability of shoot architecture in two grapevine (Vitis vinifera) cultivars. Ann Bot 2007; 99: 425-437.

20 Lebon E, Pellegrino A, Tardieu F, Lecoeur J. Shoot development in grapevine (Vitis vinifera) is affected by the modular branching pattern of the stem and intra- and inter-shoot trophic competition. Ann Bot 2004; 93: 263-274.

21 Cousins P, Johnston D, Switras-Meyer S, Meyer C. Genetic control of tendril distribution in a grapevine rootstock hybrid population. Acta Hortic 2009; 827: 337-339.

22 Cousins P, Switras-Meyer S, Vidmar J, Boyden L, Johnston D. Segregation of tendril distribution patterning in grapevine populations. Acta Hortic 2005; 689: 541-544.

23 Cousins P, Zhong G-Y. Hybrid and selfed seedling progenies of Vitis hybrid 'Roger's Red' grape segregate for tendril distribution. Acta Hortic 2014; 1082: 373-378.

24 Dangl GS, Raiche R, Sim S, Yang J, Golino DA. Genetic composition of the ornamental grape Roger's Red. Am J Enol Vitic 2010; 61: 266-271.

25 Wang L, Si Y, Dedow LK, Shao Y, Liu P, Brutnell TP. A low-cost library construction protocol and data analysis pipeline for illumina-based strand-specific multiplex RNA-seq. PLOS ONE 2011; 6: e26426.

26 Zhong S, Joung JG, Zheng Y et al. High-throughput illumina strand-specific RNA sequencing library preparation. Cold Spring Harb Protoc 2011; 2011: 940-949.

27 Andrews S. FastQC: a quality control tool for high throughput sequence data. Babraham Bioinforma 2010. Available at http://www.bioinformatics.babraham.ac. uk/projects/ (accessed Dec 2016).

$28 \mathrm{Li} \mathrm{H}$, Handsaker B, Wysoker A et al. The sequence alignment/map format and SAMtools. Bioinformatics 2009; 25: 2078-2079.

29 Wysoker A, Tibbetts K, Fennell T Picard tools version 1.90. Available at http:// picard.sourceforge.net. (Accessed Dec 2016).

30 Anders S, Pyl PT, Huber W. HTSeq-A Python framework to work with highthroughput sequencing data. Bioinformatics 2015; 31: 166-169. 
31 Robinson MD, McCarthy DJ, Smyth GK. edgeR: a Bioconductor package for differential expression analysis of digital gene expression data. Bioinformatics 2010; 26: 139-140.

32 Joung J-G, Corbett AM, Fellman SM et al. Plant MetGenMAP: an integrative analysis system for plant systems biology. Plant Physiol 2009; 151: 1758-1768.

33 Supek F, Bošnjak M, Škunca N, Šmuc T. Revigo summarizes and visualizes long lists of gene ontology terms. PLOS ONE 2011; 6: e21800; doi:10.1371/journal.pone.0021800.

34 Guo AY, Chen X, Gao G et al. PlantTFDB: a comprehensive plant transcription factor database. Nucleic Acids Res 2008; 36: D966-D969.

35 Zhang B, Li H, Riggins RB et al. Differential dependency network analysis to identify condition-specific topological changes in biological networks. Bioinformatics 2009; 25: 526-532.

36 Tian Y, Zhang B, Hoffman EP et al. KDDN: an open-source Cytoscape app for constructing differential dependency networks with significant rewiring. Bioinformatics 2015; 31: 287-289.

37 Yeung N, Cline MS, Kuchinsky A, Smoot ME, Bader GD. Exploring biological networks with cytoscape software. Curr. Protoc. Bioinforma. 2008; doi:10.1002/ 0471250953.bi0813s23.

38 Nowak K, Wójcikowska B, Gaj MD. ERF022 impacts the induction of somatic embryogenesis in Arabidopsis through the ethylene-related pathway. Planta 2015; 241: 967-985.

39 Pourcel L, Routaboul JM, Kerhoas L, Caboche M, Lepiniec L, Debeaujon I. Transparent Testa10 encodes a laccase-like enzyme involved in oxidative polymerization of flavonoids in Arabidopsis seed coat. Plant Cell 2005; 17: 2966-2980.

40 Gaufichon L, Reisdorf-Cren M, Rothstein SJ, Chardon F, Suzuki A. Biological functions of asparagine synthetase in plants. Plant Sci 2010; 179: 141-153.

41 Hwang IS, An SH, Hwang BK. Pepper asparagine synthetase 1 (CaAS1) is required for plant nitrogen assimilation and defense responses to microbial pathogens. Plant J 2011; 67: 749-762.

42 Weingartner $\mathrm{M}$, Criqui $\mathrm{M}-\mathrm{C}$, Mészáros $\mathrm{T}$ et al. Expression of a nondegradable cyclin B1 affects plant development and leads to endomitosis by inhibiting the formation of a phragmoplast. Plant Cell 2004; 16: 643-657.

43 Kitsios G, Doonan JH. Cyclin dependent protein kinases and stress responses in plants. Plant Signal Behav 2011; 6: 204-209.

$44 \mathrm{Xu}$ J, Wang XY, Guo WZ. The cytochrome P450 superfamily: key players in plant development and defense. J Integr Agric 2015; 14: 1673-1686.

45 Fernandez J, Marroquin-Guzman M, Nandakumar R et al. Plant defence suppression is mediated by a fungal sirtuin during rice infection by Magnaporthe oryzae. Mol Microbiol 2014; 94: 70-88.

46 Sels J, Mathys J, De Coninck BMA, Cammue BPA, De Bolle MFC. Plant pathogenesis-related (PR) proteins: a focus on PR peptides. Plant Physiol Biochem 2008; 46: 941-950.

47 Mohr PG, Cahill DM. Suppression by ABA of salicylic acid and lignin accumulation and the expression of multiple genes, in Arabidopsis infected with Pseudomonas syringae pv. tomato. Funct Integr Genomics 2007; 7: 181-191.

48 Machado CR, Costa de Oliveira RL, Boiteux S, Praekelt UM, Meacock PA, Menck CFM. Thi1, a thiamine biosynthetic gene in Arabidopsis thaliana, complements bacterial defects in DNA repair. Plant Mol Biol 1996; 31: 585-593.

49 Oh Y, Baldwin IT, Galis I. A Jasmonate ZIM-domain protein NaJAZd regulates floral jasmonic acid levels and counteracts flower abscission in nicotiana attenuata plants. PLOS ONE 2013; 8: e57868.

50 Lauber MH, Waizenegger I, Steinmann T et al. The Arabidopsis KNOLLE protein is a cytokinesis-specific syntaxin. J Cell Biol 1997; 139: 1485-1493.

51 Benschop JJ, Costa de Oliveira RL, Boiteux S, Praekelt UM, Meacock PA, Menck CFM. Quantitative phosphoproteomics of early elicitor signaling in Arabidopsis. Mol Cell Proteomics 2007; 6: 1198-1214.

52 Gilroy S, Suzuki N, Miller G et al. A tidal wave of signals: calcium and ROS at the forefront of rapid systemic signaling. Trends Plant Sci 2014; 19: 623-630.

53 Ho LHM, Giraud E, Lister R et al. Characterization of the regulatory and expression context of an alternative oxidase gene provides insights into cyanideinsensitive respiration during growth and development. Plant Physiol 2007; 143: 1519-1533.

54 Grimplet J, Deluc LG, Tillett RL et al. Tissue-specific mRNA expression profiling in grape berry tissues. BMC Genomics 2007; 8: 187.

55 Mizoi J, Shinozaki K, Yamaguchi-Shinozaki K. AP2/ERF family transcription factors in plant abiotic stress responses. Biochim Biophys Acta-Gene Regul Mech 2012; 1819: 86-96.

56 Hammes UZ, Nielsen E, Honaas LA, Taylor CG, Schachtman DP. AtCAT6, a sinktissue-localized transporter for essential amino acids in Arabidopsis. Plant $J$ 2006; 48: 414-426.

57 Dombrecht B, Xue GP, Sprague SJ et al. MYC2 differentially modulates diverse jasmonate-dependent functions in Arabidopsis. Plant Cell 2007; 19: 2225-2245.

58 Nakamichi N, Kita M, Ito S, Yamashino T, Mizuno T. PSEUDO-RESPONSE REGULATORS, PRR9, PRR7 and PRR5, together play essential roles close to the circadian clock of Arabidopsis thaliana. Plant Cell Physiol 2005; 46: 686-698.
59 Pumplin N, Voinnet O. RNA silencing suppression by plant pathogens: defence, counter-defence and counter-counter-defence. Nat Rev Microbiol 2013; 11: 745-760.

60 Vera-Sirera F, Gomez MD, Perez-Amador MA. DELLA Proteins, a Group of GRAS Transcription Regulators that Mediate Gibberellin Signaling. In: Gonzalez DH (ed). Plant Transcription Factors: Evolutionary, Structural and Functional Aspects. Elsevier: Universidad Nacional del Litoral, Santa Fe, Argentina, 2015, pp 313-328.

61 Saze H, Shiraishi A, Miura A, Kakutani T. Control of genic DNA methylation by a jmjC domain-containing protein in Arabidopsis thaliana. Science 2008; 319: 462-465.

62 Czemmel S, Stracke R, Weisshaar B et al. The grapevine R2R3-MYB transcription factor VvMYBF1 regulates flavonol synthesis in developing grape berries. Plant Physiol 2009; 151: 1513-1530.

63 Treutter D. Significance of flavonoids in plant resistance and enhancement of their biosynthesis. Plant Biol 2005; 7: 581-591.

64 Lauvergeat V, Decendit A, Richard T, Deluc L. Characterization of a grapevine R2R3-MYB transcription factor that regulates the phenylpropanoid pathway. Plant Physiol 2006; 140: 499-511.

65 Speyer G, Kiefer J, Dhruv H, Berens M, Kim S. Knowledge-assisted approach to identify pathways with differential dependencies. Pac Symp Biocomput 2016; 21: $33-44$.

66 Nakano T, Suzuki K, Fujimura T, Shinshi H. Genome-wide analysis of the ERF gene family. Plant Physiol 2006; 140: 411-432.

67 Iwase A, Mitsuda N, Koyama T et al. The AP2/ERF transcription factor WIND1 controls cell dedifferentiation in Arabidopsis. Curr Biol 2011; 21: 508-514.

68 So H-A, Choi SJ, Chung E, Lee J-H. Molecular characterization of stress-inducible PLATZ gene from soybean (Glycine max L.). POJ 2015; 8: 479-484.

$69 \mathrm{Li}$ S. The Arabidopsis thaliana TCP transcription factors: a broadening horizon beyond development. Plant Signal Behav 2015; 10: e1044192.

70 Yang F, Bui HT, Pautler $\mathrm{M}$ et al. A maize glutaredoxin gene, abphyl2, regulates shoot meristem size and phyllotaxy. Plant Cell Online 2015; 27: 121-131.

71 Hofmann NR. Glutaredoxin functions in floral development. Plant Cell Online 2009; 21: 363.

72 Marín-de la Rosa N, Sotillo B, Miskolczi P et al. Large-scale identification of gibberellin-related transcription factors defines group VII ETHYLENE RESPONSE FACTORS as functional DELLA partners. Plant Physiol 2014; 166: 1022-1032.

73 Maris C, Dominguez C, Allain FHT. The RNA recognition motif, a plastic RNAbinding platform to regulate post-transcriptional gene expression. FEBS J 2005; 272: 2118-2131.

74 Coca M, San Segundo B. AtCPK1 calcium-dependent protein kinase mediates pathogen resistance in Arabidopsis. Plant J 2010; 63: 526-540.

$75 \mathrm{Wu}$ Y, Deng Z, Lai J et al. Dual function of Arabidopsis ATAF1 in abiotic and biotic stress responses. Cell Res 2009; 19: 1279-1290.

76 Mazzucotelli E, Mastrangelo AM, Crosatti C, Guerra D, Stanca AM, Cattivelli L. Abiotic stress response in plants: when post-transcriptional and posttranslational regulations control transcription. Plant Sci 2008, 174: 420-431.

77 Aubert D, Chen L, Moon YH et al. EMF1, a novel protein involved in the control of shoot architecture and flowering in Arabidopsis. Plant Cell 2001; 13: 1865-1875.

78 Fernandez-Perez F, Pomar F, Pedreo MA, Novo-Uzal EN-U. The suppression of AtPrx52 affects fibers but not xylem lignification in Arabidopsis by altering the proportion of syringyl units. Physiol Plant 2015; 154: 395-406.

79 Tal I, Zhang Y, Jørgensen ME et al. The Arabidopsis NPF3 protein is a GA transporter. Nat Commun 2016; 7: 11486

80 Pike S, Gao F, Kim MJ, Kim SH, Schachtman DP, Gassmann W. Members of the NPF3 transporter subfamily encode pathogen-inducible nitrate/nitrite transporters in grapevine and Arabidopsis. Plant Cell Physiol 2014; 55: 162-170.

81 Tuteja N. Signaling through G protein coupled receptors. Plant Signal Behav 2009; 4: 942-947.

82 Palatnik JF, Allen E, Wu X et al. Control of leaf morphogenesis by microRNAs. Nature 2003; 425: 257-263.

83 Koyama T, Furutani M, Tasaka M, Ohme-Takagi M. TCP transcription factors control the morphology of shoot lateral organs via negative regulation of the expression of boundary-specific genes in Arabidopsis. Plant Cell Online 2007; 19: 473-484.

84 Sugio A, MacLean AM, Hogenhout SA. The small phytoplasma virulence effector SAP11 contains distinct domains required for nuclear targeting and CIN-TCP binding and destabilization. New Phytol 2014; 202: 838-848.

85 Czemmel S, Heppel SC, Bogs J. R2R3 MYB transcription factors: key regulators of the flavonoid biosynthetic pathway in grapevine. Protoplasma 2012; 249: 109-118.

86 Matus JT, Aquea F, Arce-Johnson P. Analysis of the grape MYB R2R3 subfamily reveals expanded wine quality-related clades and conserved gene structure organization across Vitis and Arabidopsis genomes. BMC Plant Biol 2008; 8: 83.

87 Li S, Yin X-R, Xie X et al. The citrus transcription factor, CitERF13, regulates citric acid accumulation via a protein-protein interaction with the vacuolar proton pump, CitVHA-c4. Sci Rep 2016; 6: 20151 
88 Feller A, MacHemer K, Braun EL, Grotewold E. Evolutionary and comparative analysis of MYB and bHLH plant transcription factors. Plant J 2011; 66: 94-116.

89 Li S. Transcriptional control of flavonoid biosynthesis: fine-tuning of the MYBbHLH-WD40 (MBW) complex. Plant Signal Behav 2014; 9: e27522.

90 Satoh R, Fujita Y, Nakashima K, Shinozaki K, Yamaguchi-Shinozaki K. A novel subgroup of bZIP proteins functions as transcriptional activators in hypoosmolarityresponsive expression of the ProDH gene in Arabidopsis. Plant Cell Physiol 2004; 45: 309-317.

91 Boss PK, Thomas MR. Association of dwarfism and floral induction with a grape 'green revolution' mutation. Nature 2002; 416: 847-850.

92 Lacroix CR, Posluszny U. Phyllotactic patterns in some members of the Vitaceae. Bot Gaz 1989; 150: 303-313.

93 Wilson T, Posluszny U. Complex tendril branching in two species of Parthenocissus: implications for the vitaceous shoot architecture. Can J Bot Can Bot 2003; 81: 587-597.

94 Pandey S, Somssich IE. The role of WRKY transcription factors in plant immunity. Plant Physiol 2009; 150: 1648-1655.

95 Jiang Y, Deyholos MK. Comprehensive transcriptional profiling of $\mathrm{NaCl}$-stressed Arabidopsis roots reveals novel classes of responsive genes. BMC Plant Biol 2006; 6: 25

96 Hofer J, Turner L, Moreau C et al. Tendril-less regulates tendril formation in pea leaves. Plant Cell 2009; 21: 420-428.

97 Mizuno S, Sonoda M, Tamura $Y$ et al. Chiba tendril-less locus determines tendril organ identity in melon (Cucumis melo L.) and potentially encodes a tendrilspecific TCP homolog. J Plant Res 2015; 128: 941-951.

98 Matus JT, Aquea F, Espinoza C et al. Inspection of the grapevine BURP superfamily highlights an expansion of RD22 genes with distinctive expression features in berry development and ABA-mediated stress responses. PLOS ONE 2014; 9: e110372.

99 Leyser HMO, Lincoln CA, Timpte C, Lammer D, Turner J, Estelle M. Arabidopsis auxin-resistance gene AXR1 encodes a protein related to ubiquitin-activating enzyme E1. Nature 1993; 364: 161-164.

100 Yin R, Han K, Heller W et al. Kaempferol 3-O-rhamnoside-7-O-rhamnoside is an endogenous flavonol inhibitor of polar auxin transport in Arabidopsis shoots. New Phytol 2014; 201: 466-475.

101 Li S, Zachgo S. TCP3 interacts with R2R3-MYB proteins, promotes flavonoid biosynthesis and negatively regulates the auxin response in Arabidopsis thaliana. Plant J 2013; 76: 901-913.

102 Li C, Potuschak T, Colón-Carmona A, Gutiérrez RA, Doerner P. Arabidopsis TCP20 links regulation of growth and cell division control pathways. Proc Natl Acad Sci USA 2005; 102: 12978-12983.

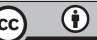

This work is licensed under a Creative Commons Attribution 4.0 International License. The images or other third party material in this article are included in the article's Creative Commons license, unless indicated otherwise in the credit line; if the material is not included under the Creative Commons license, users will need to obtain permission from the license holder to reproduce the material. To view a copy of this license, visit http://creativecommons.org/licenses/ by/4.0/

(c) The Author(s) 2017

Supplementary Information for this article can be found on the Horticulture Research website (http://www.nature.com/hortres) 